\title{
Determinación de huevos de Toxocara canis en suelo de tres parques públicos de Duitama (Boyacá)
}

\section{Toxocara canis eggs determination in Duitama (Boyacá) three public parks soil}

Fecha recepción: 4 de agosto de 2015

Fecha aceptación: 1 de diciembre de 2015

Camilo Ernesto Guarín-Patarroyo ${ }^{1}$ Marly Julieth Serrato ${ }^{2}$

Fabián Rodrigo Sánchez-Cuervo ${ }^{3}$

\section{Resumen}

Toxocara canis es un nemátodo frecuente en el intestino delgado de los perros, que se infestan a través de migración transplacentaria, vía galactógena o por ingestión de huevos embrionados; infecta también a las personas, principalmente a menores de cinco años, produciendo la toxocariasis humana, zoonosis catalogada como desatendida; se adquiere especialmente por ingesta de tierra contaminada y verduras sin adecuado manejo sanitario, y causa enfermedades como el Síndrome de Larva Migrans visceral y cutánea y Toxocariasis ocular. Este estudio tuvo como objetivo determinar, mediante la técnica de Sloss, la presencia de huevos de Toxocara canis en suelos de tres parques públicos de Duitama, y, además, identificar los mecanismos de trasmisión de la Toxocara canis en la población que acude a estos parques. Para ello se colectaron 76 muestras de suelo de $10 \mathrm{~cm}$. de largo por $10 \mathrm{~cm}$. de ancho y 3 de profundidad, las cuales fueron repartidas en dos estratos y almacenadas en bolsas plásticas herméticas para su posterior estudio en el laboratorio. Los resultados obtenidos indican que los huevos del parásito se encontraron en el $34.7 \%$ de las muestras recolectadas, señalando un nivel de riesgo para la población. De acuerdo con las variables $\mathrm{pH}$, humedad y estratos, no se obtuvo asociación entre estas y la identificación del huevo. La presencia de $T$. canis en los parques indica que la población de la ciudad de Duitama está expuesta al riesgo potencial de adquirir el parásito.

Palabras clave: larva migrans; salud pública; toxocariasis; zoonosis.

1 M.Sc. Fundación Universitaria Juan de Castellanos (Tunja-Colombia). cguarin@jdc.edu.co.

2 Profesional Independiente.

3 Esp. Fundación Universitaria Juan de Castellanos (Tunja-Colombia). fsanchez@jdc.edu.co. 


\begin{abstract}
Toxocara canis is an Ascarideo nematode, common in the small intestine of dogs, which become infected through transplacental migration by galactogen way, or embryonated eggs ingestion. It also infects humans, mainly affects children under five years old, producing human toxocariasis, and classified as a neglected zoonosis. Infestation is especially spread by ingestion of contaminated soil and vegetables without adaquated health management, and causing illiness such as Migratory Syndrome Visceral Larva, plus dermal and ocular toxocariasis. The aim of the study was to determine by the Sloss technique the presence of Toxocara canis eggs in Duitama three public parks soils, besides to identify the Toxocara canis transmission mechanisms in the population that goes to these parks. For that purpose, were collected 76 samples from the ground, $10 \mathrm{~cm}$ long, by $10 \mathrm{~cm}$ wide, and $3 \mathrm{~cm}$ depth, then stored in sealed plastic bags, for a later study at the laboratory. The results indicate that parasite eggs were found in $34.7 \%$ of the samples collected, ponting a risk level to the population. According to Variables $\mathrm{pH}$, humidity and strata, no association between them and the identification of the egg was obtained. Nevertheless, the presence of T. canis in the parks indicates that the population of the city of Duitama is exposed to the serious potential risk of acquiring the parasite.
\end{abstract}

Keywords: larva migrans; public health; toxocariasis; zoonosis. 


\section{Introducción}

Toxocara canis es un helminto de distribución mundial que causa la toxocariasis, enfermedad zoonótica muy común, debido a la profusa contaminación del medio con huevos de este parásito, especialmente en áreas públicas y patios de casas particulares (1). Toxocora canis se desarrolla principalmente en el intestino delgado del perro, de donde migra por el torrente sanguíneo a pulmón e hígado (2), causando, principalmente: apatía, inapetencia, debilidad, obstrucción en las vías biliares, diarrea, vómito y sangre en las heces (4); este parásito pertenece al género Toxocara, de la familia Ascaridae, de cuyas especies las de mayor importancia son Toxocara canis y Toxocara cati, por su riesgo zoonótico (3).

La afectación multisistémica producida por la toxocariasis, que afecta principalmente a jóvenes y niños, constituye un problema sanitario ampliamente difundido en todo el mundo (5), y un problema de salud pública poco atendido a nivel mundial. En los seres humanos, los síntomas más comunes de la toxocariasis son: manifestaciones pulmonares, dolor abdominal, anorexia, hepatomegalia y trastornos neuronales y oculares (6).

Según barriga 1988 el 99,4\% de los perros recién nacidos están infectados, al igual que el $40 \%$ de los perros menores de 6 meses (7). En Colombia, en un estudio realizado en el 2005, se encontró una prevalencia de $2.5 \%$ de perros afectados por T. canis (8); en Boyacá se han desarrollado varios estudios, uno de los cuales detectó, para la ciudad de Tunja, la presencia de nemátodos en el $74 \%$ de las muestras de materia fecal de perros y de los suelos de diferentes parques de esta ciudad, y de protozoarios en el $88 \%$ (9).

El propósito de esta investigación fue identificar la presencia o no de huevos del parásito en los tres parques públicos más concurridos de la ciudad de Duitama, relacionando su presencia con la humedad y el pH del suelo e identificando los mecanismos de transmisión en la población que acude a estos parques.

\section{Materiales y métodos}

El estudio es epidemiológico descriptivo observacional de corte transversal, en el cual se utilizó la técnica de Sloss para identificar los huevos de $T$. canis. Se realizó mediante el método de muestreo aleatorio estratificado, tomando los estratos tierra y sombra, en los parques Recreacional del Norte, el Carmen y Simón Bolívar. La muestra se determinó por medio de la fórmula para el cálculo de poblaciones finitas; la población se tomó como el total de centímetros cuadrados que miden los tres parques por muestrear; en total fueron $784.990 \mathrm{~cm}^{2} \mathrm{y}$ una $\mathbf{n}$ de muestra de 76 , que se repartieron en los dos estratos para el estudio.

Se tomaron muestras de tierra, segmentos de tierra y pasto en constante sombra, para ello se araron grillas de $4 \mathrm{~m}^{2}$ (10), las cuales no pueden ser áreas con cemento. Las muestras fueron de $10 \mathrm{~cm}$. de largo por $10 \mathrm{~cm}$. de ancho y 3 de profundidad; cada muestra fue guardada en bolsas herméticas y refrigeradas a 4 grados para conservar sus características hasta su procesamiento dentro de 72 horas (11).

Para determinar la humedad del suelo, se tomó como referencia el método del tacto, que establece tomar una porción de tierra y exprimirla con la palma de la mano para medir aproximadamente la humedad en el suelo (12). Para este estudio se tomarán como referencia tres clases de humedad (leve, moderada y alta).

Se aplicaron 90 encuestas a los visitantes de los parques y se realizó observación directa sobre los hábitos de las personas que Ilevan mascotas y niños al parque.

Se utilizó la prueba de Chi-cuadrado para determinar la relación entre la presencia o ausencia del parásito y el parque, así como la asociación de las variables de $\mathrm{pH}$ y humedad. La encuesta, la observación y la literatura sobre mecanismos de transmisión son triangulados utilizando una matriz en Exce ${ }^{\circledR}$ por cada tema de importancia como factor de riesgo para la contaminación por Toxocara canis, permitiendo relacionar cada tema dentro de 
los tres métodos de obtención de información y analizarlo de manera cualitativa.

\section{Resultados y discusión}

En el laboratorio se encontraron huevos larvados de Toxocara canis en 25 muestras, que represen- tan el $34,7 \%$ del total analizado, lo cual indica un nivel medio de riesgo para la zona. El número de muestras positivas fue similar entre los parques estudiados. La proporción de presencia en los parques Recreacional del Norte y Simón Bolívar (12,5 $\%$, y en el parque El Carmen la prevalencia fue más baja $(9,7 \%)$, (Tabla I).

Tabla I. Presencia de huevos de Toxacara canis en los parques de Duitama.

\begin{tabular}{|l|c|c|c|c|c|}
\hline Parque & Negativo & & Positivo & & Total \\
\hline Recreacional del norte & 14 & $19,4 \%$ & 9 & $12,5 \%$ & 23 \\
\hline El Carmen & 17 & $23,6 \%$ & 7 & $9,72 \%$ & 24 \\
\hline Simón Bolívar & 16 & $22,22 \%$ & 9 & $12,5 \%$ & 21 \\
\hline Total & 47 & $63.3 \%$ & 25 & $34,7 \%$ & 72 \\
\hline
\end{tabular}

Los parques públicos y las zonas de juegos se han convertido en zonas de adquisición de la toxocariasis, por tal razón, alrededor del mundo se han realizado diferentes estudios que señalan la presencia de Toxocara canis (5). Gracias a este estudio, que señala que el $34,7 \%$ de las muestras $(n=72)$ contienen huevos de Toxocara canis en los tres parques, se indica que los parques públicos de la ciudad de Duitama constituyen sitios de riesgo de infección con huevos de $T$. canis para las personas que concurren a ellos. Estos resultados de contaminación de T. canis son similares a los del estudio realizado por Romero et al. (13), quienes encontraron en el total de los parques estudiados el 30,3\% de presencia; cifras que son altas en relación con otros estudios, como el de Polo et al. (14) en Suba, Colombia, y el de Young et al. (15) en Lima, Perú, quienes obtuvieron datos menores de $8 \%$ de presencia. Estos resultados son de gran importancia, ya que sin importar que la proporción sea baja, está indicando la presencia, lo cual ya se convierte en problema de salud pública.

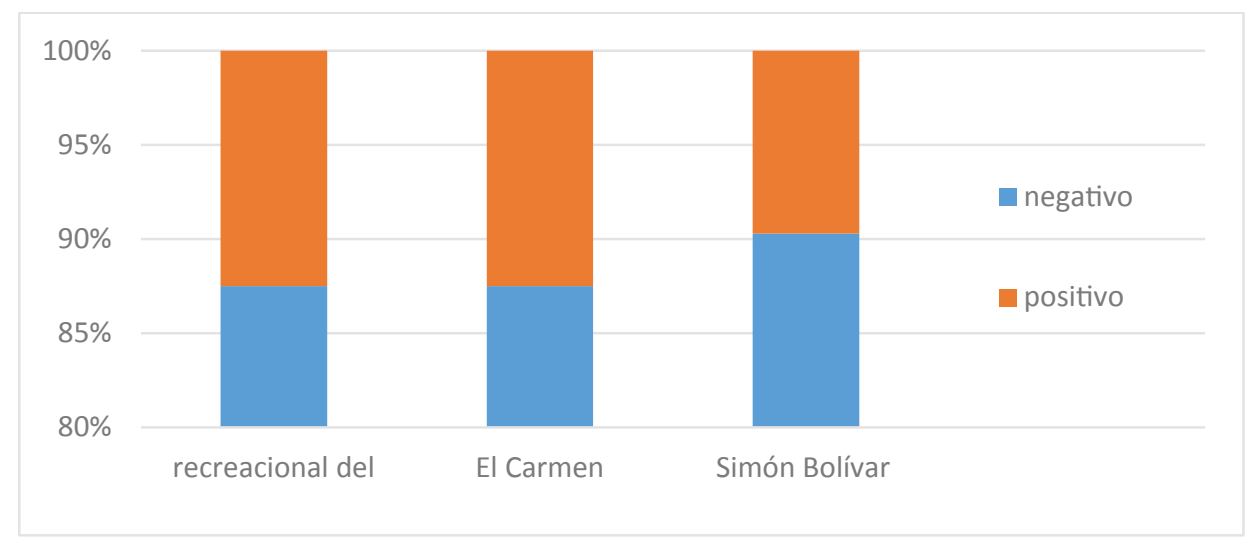

Figura 1. Presencia de huevos de Toxacara canis en los parques de Duitama.

La proporción de presencia de T. canis encontrada en este estudio se puede considerar baja, en comparación con los resultados de investigaciones similares en otras regiones y el mundo. Reyes (1) señala que la presencia de huevo de este parásito es del $88.3 \%$ en lugares públicos y casas particulares en la ciudad de Valdivia, Chile. En São Paulo, Brasil, se encontró el $90.6 \%$ de huevos en parques 
públicos, según Santarém et al. (16); y en plazas públicas de Concordia, Brasil, se identificó el 28 $\%$ de huevos de $T$. canis, según estudio de Lettieri et al. (17). Otros estudios realizados indican el 53 $\%$ de presencia en plazas y parques de Asunción, Paraguay, según Canese et al. (3), y el $52.08 \%$ en parques recreacionales en Trujillo, según Goicochea (18).

Para ver posibles asociaciones entre la presencia de la Toxocara canis y los parques del estudio, se realizó una prueba de Chi-cuadrado, con un nivel de significancia de 0,05 y tres grados de libertad, obteniendo $X 2=15,11$, siendo este resultado mayor que el valor crítico del Chi-cuadrado $(X 2=7,81)$, encontrando dependencia entre la presencia del parásito y los parques.

En el caso del grupo sombra, la prueba de Chi-cuadrado, con nivel de significancia de 0.05 y tres grados de libertad, tuvo como resultado $\mathrm{X} 2=2.3$, interpretándose como que no existe relación entre la detección del parásito en cada parque y la condición sombra, similar a lo visto con el sustrato tierra.

Al revisar si existía asociación entre la presencia/ ausencia de la Toxocara y el grupo tierra y el grupo sombra, se obtuvo, para un nivel de confianza del 0.05 y dos grados de libertad, el valor de $\mathrm{X} 2=0.94$, corroborando que no existe asociación entre los grupos (tierra y sombra) y la presencia del parásito.

Se comprobó la varianza de las medias por medio de una prueba $\mathrm{F}$ con una confiabilidad del 0.05, 11 grados de libertad, $\mathrm{F}=4.77$ y $\mathrm{P}=0.007$, por lo que hay diferencias significativas entre las medias de los dos $\mathrm{pH}$ entre los grupos tierra y sombra del Parque Recreacional.

La varianza de las medias para el Parque Simón Bolívar en la prueba $\mathrm{F}$, con una confiabilidad del $0.05,11$ grados de libertad, mostró un $F=0.14$ y $\mathrm{P}=0.01$, por lo que hay diferencias significativas entre las medias de los dos $\mathrm{pH}$ entre los grupos tierra y sombra.

La humedad se midió como leve, moderada y alta.
En ninguno de los parques ni por estrato se encontró humedad leve; por tanto, se buscó asociación entre los estratos y la humedad encontrada. Para esto se realizó una prueba de Chi-cuadrado, con una confiabilidad de 0.05 y dos grados de libertad; se obtuvo X2 $=0,64$, interpretándose que no existe asociación.

En el Parque El Carmen, la varianza de las medias del $\mathrm{pH}$ entre los grupos tierra y sombra, según la prueba $F$, no tuvo diferencias significativas (confiabilidad del 0,05, 11 grados de libertad, mostró un $\mathrm{F}=1.15$ y $\mathrm{P}=0.41$ ). Se revisó la asociación entre el $\mathrm{pH}$ y la presencia/ausencia de la Toxocara canis por medio de un Chi-cuadrado. El resultado de este análisis, con una confiabilidad del 0,05 y 2 grados de libertad, fue de $X 2=<0,01$, interpretándose que la presencia del parásito no se asocia al $\mathrm{pH}$ de los dos grupos estudiados.

Existen investigaciones que asocian la presencia de huevos de Toxocara canis con el tipo de suelo, que señalan que la textura del suelo influye. Salinas et al. (20) encontraron mayor presencia de huevos en el suelo arcillo-lodoso, y Forey considera que estos suelos ofrecen condiciones óptimas de humedad para la viabilidad y desarrollo de los huevos (21). En otras investigaciones no se obtuvo relación entre la presencia de este parásito y el tipo del suelo; según lannacone et al. (22), en Lima Perú, no se observó diferencias entre la presencia de huevos en el césped y en el suelo de los parques públicos estudiados; en la ciudad de Coro, Venezuela, Cazorla et al. (23) tampoco encontraron relación entre el tipo de suelo y la presencia de huevos de T.canis.

En este estudio se corrobora la presencia de este parásito en áreas verdes de parques públicos, pero también se demuestra que no existe ninguna relación entre las variables sombra y tierra en las muestras, similar al estudio de Gallardo y Camacho (24), que demuestran que los huevos de T. canis son muy resistentes al medioambiente y pueden localizarse en la tierra, pasto y sombra de patios y jardines. Estos resultados son atribuidos a que los parques muestreados tienen áreas de vegetación y lugares de sombra que proporcionan factores que favorecen la sobrevivencia de los huevos, además 
de tener un gran número de visitantes y, con ellos, mascotas (Loza, 2006). (25)

Se ha demostrado que la supervivencia de formas infectivas se encuentra asociada a las condiciones de humedad, temperatura y $\mathrm{pH}$ del ambiente; los huevos de Toxacara canis tienen una alta viabilidad en suelos húmedos y climas templados, y son incapaces de sobrevivir y desarrollarse o persistir en el suelo por tiempos prologados en condiciones extremas de estos factores (Pierangel et al., 2003) (26). Existen varios estudios que describen la presencia de huevos de T.canis en parques públicos, sin embargo, pocos relacionan la aparición del parásito con las condiciones ambientales.

En este estudio, el $\mathrm{pH}$ no resultó ser una variable significativa asociada a la presencia de huevos de Toxocara canis en los suelos de los parques públicos de la ciudad de Duitama, al igual que los estudios de lannacone et al. (21) y Cazorla et al. (22), los cuales tampoco encontraron relación entre las variaciones de $\mathrm{pH}$ de los suelos y la presencia de huevos de $T$. canis.

Dentro de las condiciones óptimas para la viabilidad de este parásito se encuentra la temperatura óptima, unida a la humedad relativa adecuada; según la literatura, el parásito $T$. canis puede sobrevivir entre los 5 y los $38{ }^{\circ} \mathrm{C}$, junto con una humedad relativa elevada (12); estas cifras demuestran que el parásito tiene alto margen de tolerancia, que lo convierte en un problema de salud pública. Los resultados de la humedad en los suelos analizados en Duitama señalan condiciones óptimas para la viabilidad de este parásito, pues en la mayoría de las muestras la humedad relativa era moderada y alta; pero estadísticamente, los datos señalan que no existe asociación ni relación entre esta y la presencia de los huevos de $T$. canis, similar al estudio realizado por Castillo Y. et al (27), y al de Sommerfelt et al. (28). Duitama cuenta con los parques en buen estado, con áreas con bastante vegetación, la cual crea condiciones de humedad y temperatura óptimas para la supervivencia de los huevos de Toxocara canis (29)

\section{Conclusiones}

La presencia de Toxocara canis en los parques públicos de la ciudad de Duitama representa un riesgo de zoonosis y salud pública para a las personas que acuden a estos sitios de esparcimiento familiar, sobre todo si los niños juegan en el suelo, con la tierra o con objetos contaminados, ya que muchos de los propietarios de mascotas y la ciudadanía en general no están educados sobre el modo de transmisión de esta parasitosis a través del contacto de tierra infectada (3).

No hay relación entre la presencia de huevos y el estrato estudiado de tierra y sombra, igualmente con las variables físico-químicas del suelo (humedad y $\mathrm{pH}$ ).

El principal factor asociado a la contaminación de parques por huevos de Toxocara spp. lo constituyen las excretas de los perros, por lo que debe establecerse un programa de desparasitación de las mascotas, aunado a prácticas de higiene y prevención en los niños, Y espacios especiales para las mascotas, que no sean los mismos de los niños; los parques para las familias no deben ser los sanitarios de los perros, pues así los propietarios alcen las heces, el suelo se contamina. Así mismo, el incumplimiento de las normas de salud pública vigentes por parte de los propietarios de las mascotas que no recogen las heces es uno de los principales factores para la contaminación de los parques públicos.

\section{Referencias}

(1) Reyes Y. Determinación del riesgo de infección con huevos de Toxocara canis en lugares públicos y patios de casas particulares en la ciudad de Valdivia. 2008.

(2) Martínez I., Fernández A., Vázquez T., O Ruiz A. Frecuencia de Toxocora canis en perros y áreas verdes del sur de la ciudad de México, Distrito Federal. Vet. Mex., 1999; 29 (3): 239244.

(3) Canese A., Domínguez R., Otto C., Ocampos C., Mendonca E. Huevos infectivos de toxo- 
cara, en arenas de plazas y parques de Asunción, Paraguay. Rev. chil. pediatr., 2003; 74 (6): 611-616. DOI: http://dx.doi.org/10.4067/ S0370-41062003000600010.

(4) Juanquera P. Parasitipedia.net. (en línea) 2013 (fecha de acceso 29 de junio 2015): Disponible en: http://parasitipedia.net/index. php?option $=$ com_content $\&$ view $=$ article\&id $=1460$ \&ltemid $=1591$.

(5) Despommier D. Toxocariasis: clinical aspects, epidemiology, medical ecology, and molecular aspects. Clin Microbiol Rev, 2003; 16(2): 265-272. DOI: http://dx.doi.org/10.1128/ CMR.16.2.265-272.2003.

(6) Uribarren T. Toxocariasis (en línea) 2011 (fecha de acceso 2 de julio): Disponible en: http://www.facmed.unam.mx/deptos/microbiologia/parásitologia/larva-migrans-visceral. html.

(7) Sánchez U. Determinación de la presencia de huevos de Toxocara canis en suelo de los parques públicos del municipio de Garagoa, Boyacá. Tunja: Fundación Universitaria Juan de Castellanos. 2014.

(8) Téllez A. Evaluación de la contaminación con Cryptosporidium spp. en suelos de los principales parques públicos y zonas verdes de la ciudad de Tunja. Tunja: Fundación Universitaria Juan de Castellanos. 2012.

(9) Vásquez S. Comparación de tres sistemas de muestreo para determinar la contaminación del suelo de áreas públicas de la ciudad de Valdivia con huevos de Toxocara canis. Universidad Austral de Chile. 2007.

(10) Martín E. Métodos para medir la humedad del suelo para la programación del Riego. ¿Cuándo? Arizona Cooperative Extension. 2010.

(11) Hernández L. Toxocariasis. (en línea) 2010 (fecha de acceso 15 de junio de 2015): Disponible en: https://es.scribd.com/doc/34265507/ TOXOCARIASIS.
(12) Delgado O. Rodríguez-Morales, J. Aspectos clínico-epidemiológicos de la toxocariasis: una enfermedad desatendida en Venezuela y América Latina. Bol. Mal. Salud Amb. 2009; 49 (1): 1-33.

(13) Romero C., Mendoza G., Bustamante L., Crosby M., Ramírez N. Presencia y viabilidad de Toxocara spp. en suelos de parques públicos, jardines de casas y heces de perros en Nezahualcoyotl, México. Red de revistas científicas de América Latina, 2011.

(14) Polo L., Cortés J., Villamil L. Contaminación de los parques públicos de la localidad de Suba (Bogotá) con Nemátodos Zoonóticos. Revista Salud Pública, 2007; 9 (4): 550 - 557.

(15) Young C., Yauri R., Yanes S., Castro J., Vera K., Villegas J., Zúñiga, P. Zari, C. et al. Frecuencia de Toxocara sp. en los parques del distrito de Bre-a. Lima- Perú. Revista Peruana de Epidemiología, 2011; 15 (3): 1-4.

(16) Santarém V., Pereira C., Porto Alegre C. Contamination of public parks in Presidente Prudente (São Paulo, Brazil) by Toxocara spp. Eggs. Rev. Bras Parasitol Vet, 2012; 21 (3): 323-325. DOI: http://dx.doi.org/10.1590/ S1984-29612012000300029.

(17) Lettieri T., Rossi L., De Freitas L., Gaspari N., Piva S., Meneghello FA. Prevalence of Toxocara canis infection in public squares of the Concórdia City, Santa Catarina, Brazil. Parasitología Latinoamericana, 2008; 63: 6971. DOI: http://dx.doi.org/10.4067/s071777122008000100012.

(18) Goicochea A. Prevalencia de Toxocara canis en parques recreacionales del Distrito de Trujillo durante el mes de julio. Facultad de ciencias agropecuarias, escuela académico-profesional de medicina veterinaria, 2012.

(20) Salinas P., Matamala M., Schenone H. Prevalencia de hallazgo de huevos de Toxocara canis en plazas de la Región Metropolitana de la ciudad de Santiago, Chile. Bol. Chil. Parasitol, 
2001; 56 (3-4): 102-105. DOI: http://dx.doi. org/10.4067/S0365-94022001000200013.

(21) Forey TW. Veterinary Parasitology: Reference Manual, 5th Edition. Blackwell publishing. lowa. 2001.

(22) lannacone J., Alvariño L., Cárdenas J. Contaminación de los suelos con huevos de Toxocara canis en parques públicos de Santiago de Surco, Lima, Perú, 2007-2008. Asociación Peruana de Helmintología e Invertebrados Afines (APHIA). 2012.

(23) Cazorla D., Morales P., Acosta M. Contaminación de suelos con huevos de Toxocara spp. (Nematoda, Ascaridida) en parques públicos de la ciudad de Coro, Estado Falcon, Venezuela. Rev Cient. (Maracaibo), 2007; 17(2): 117-122.

(24) Gallardo Y., Camacho S. Infección por Toxocara canis y factores de riesgo en niños de la comunidad Agua Azul, estado Yyaracuy. Rev. Salud, Arte y Cuidado, 2012; 5 (1): 21-27.

(25) Loza A., González J., Marín G. Estudio epidemiológico de Toxocara sp. y Ancylostoma sp. en canes y paseos públicos de los distritos I al
V de Santa Cruz de la Sierra. Rev. Electrónica de Veterinaria, 2006; VII (9): 1-23.

(26) Pierangeli N., Giayetto A., Manacorda A., Barbieri L., Soriano S., Veronesi A. et al. Estacionalidad de parásitos intestinales en suelos periurbanos de la ciudad de Neuquén, Patagonia Argentina. Tropical Medicine and International Health, 2003; 8 (3): 259-263. DOI: http://dx. doi.org/10.1046/j.1365-3156.2003.01006.x.

(27) Castillo Y., Bazan H., Alvarado D., Sáez G. Estudio epidemiológico de Toxocara canis en parques recreacionales del distrito de San Juan de Lurigancho, Lima- Perú. Parasitol, 2001; 25 (3-4): 109-114. DOI: http://dx.doi. org/10.4067/s0716-07202001000300007.

(28) Sommerfelt I., Cardillo N., López C., Ribicich M., Gallo C., Franco A. Prevalence of Toxacara Cati and Other Parasites in Cat'S Faeces Collected from the Open Spaces of Public Institutions. Bs. As. Argentina. Elsevier, 2006.

(29) Castillo D. Contaminación ambiental por huevos de Toxocara spp en algunas plazas y parques públicos de Santiago de Chile. Bol. Chil. Parasitol., 2000; 55 (3-4): 86-91. DOI: http://dx.doi.org/10.4067/S036594022000000300010 . 